\title{
Cerebral Metabolic Changes Related to Freezing of Gait in Parkinson Disease
}

\author{
Trina Mitchell ${ }^{1,2}$, Alexandra Potvin-Desrochers ${ }^{1,2}$, Anne-Louise Lafontaine ${ }^{3}$, Oury Monchi ${ }^{4}$, Alexander Thiel ${ }^{5,6}$, \\ and Caroline Paquette ${ }^{1,2}$
}

${ }^{I}$ Department of Kinesiology and Physical Education, McGill University, Montréal, Quebec, Canada; ${ }^{2}$ Centre for Interdisciplinary Research in Rehabilitation, Montréal, Quebec, Canada; ${ }^{3}$ Montreal Neurological Institute and Hospital, McGill University, Montréal, Quebec, Canada; Movement Disorders Unit, McGill University Health Centre, Montréal, Quebec, Canada; ${ }^{4}$ Department of Clinical Neurosciences and Hotchkiss Brain Institute, University of Calgary, Calgary, Alberta, Canada; ${ }^{5}$ Department of Neurology and Neurosurgery, McGill University, Montréal, Quebec, Canada; and ${ }^{6}$ Jewish General Hospital, Lady Davis Institute for Medical Research, Montréal, Quebec, Canada

Freezing of gait (FOG) in Parkinson disease (PD) often occurs during steering of gait (i.e., complex gait), which is thought to arise from executive dysfunction. Our aim was to test whether cognitive corticobasal ganglia-thalamocortical circuitry is impaired and whether alternate neural circuits are used for complex gait in PD with FOG. Methods: Eighteen individuals with idiopathic PD in the off-medication state, 9 with FOG (aged $68 \pm 6$ y) and 9 without FOG (aged $65 \pm 5$ y), were included. PET was used to measure cerebral glucose metabolism during 2 gait tasks, steering and straight walking, performed during the radiotracer uptake period. Results: During steering, there was a reduced change in cerebral glucose metabolism within the cognitive corticothalamic circuit. More specifically, those with FOG had less activation of the posterior parietal cortex, less deactivation of the dorsolateral prefrontal cortex and thalamus, and increased activation in the supplementary motor area. Interestingly, activity in the dorsolateral prefrontal cortex correlated with gait impairment (i.e., reduced stride length) in the FOG group. Conclusion: These results demonstrate decreased parietal control and an alternate control mechanism mediated by prefrontal and supplementary motor areas in PD with FOG.

Key Words: cerebral glucose metabolism; complex gait; freezing of gait; Parkinson disease; humans

J Nucl Med 2019; 60:671-676

DOI: 10.2967/jnumed.118.218248

$\mathbf{F}$ reezing of gait (FOG) in Parkinson disease (PD) is a debilitating symptom characterized by episodic motor blocks during gait (1). FOG is most often triggered by steering of gait, which comprises most steps in daily life (2), and compared with forward walking, requires increased executive control (3). Recent efforts have probed the control of the more challenging gait in PD with FOG (4-7).

Conflict monitoring and top-down control are important features of the corticobasal ganglia-thalamic structure $(8,9)$. It is known that hyperdirect connections between the supplementary

Received Jul. 23, 2018; revision accepted Sep. 25, 2018.

For correspondence or reprints contact: Caroline Paquette, Department of Kinesiology and Physical Education, McGill University, 475 Pine Ave. W., Montreal, Quebec, H2W 1S4, Canada.

E-mail: caroline.paquette@mcgill.ca

Published online Oct. 12, 2018.

COPYRIGHT (C 2019 by the Society of Nuclear Medicine and Molecular Imaging. motor area and the subthalamic nucleus subserving these functions are impaired in FOG (10-12). Thus, it is hypothesized that impairment of frontostriatal executive circuits contributes to FOG. Another hypothesis suggests that suprathreshold activity of motor and cognitive circuits leads to cross talk of these normally segregated pathways, resulting in FOG (13). Both hypotheses may be explained by high attentional demands resulting from reduced gait automaticity. It has been proposed that internally driven motor programs are impaired in PD with FOG and ordinarily automatic tasks such as gait require increased attentional control $(10,14)$. In such cases, increased activity of frontal and parietal cortices would strongly inhibit the subcortical basal ganglia and brain stem nuclei responsible for motor output (i.e., globus pallidus internal segment and pedunculopontine nucleus), requiring increased top-down control during gait $(14,15)$.

The most frequent method to quantify gait-related whole-brain activity is functional MRI (fMRI) during which the patient performs mental imagery of gait while lying supine in the scanner. These paradigms have suggested similar involvement of cortical and subcortical substrates in FOG; however, the results are contradictory $(5,16)$. An important issue arising is that mental imagery is unable to accurately capture gait in PD with FOG because of the discrepancy between perceived and actual walking resulting from sensory impairments (17). fMRI has been used to study turning in FOG during a virtual-reality paradigm with continuous foot pedaling. Here, PD with FOG shows increased activity in the inferior frontal regions involved in a stopping network, and decreased activity in the parietal and supplementary motor areas (18). These findings were taken as evidence that individuals with FOG have a tendency toward hesitation and support hypotheses for reduced gait automaticity. Still, postural control requiring complex cortical processing is absent from these paradigms (19). More recently, PET has been used to measure whole-brain cerebral glucose metabolism, a marker of brain activity, during unconstrained motor tasks performed during the radiotracer uptake period (20).

We recently used ${ }^{18} \mathrm{~F}$-FDG PET in healthy individuals to measure regional cerebral glucose metabolism (rCGM) during steering of gait (i.e., complex walking), contrasted with steady-state forward walking (i.e., a simple walking reference task). Bilateral frontoparietal regions composing the cognitive corticobasal ganglia-thalamocortical circuitry were recruited for steering (21). Understanding changes to this type of complex control in PD with FOG could better elucidate its pathophysiology. Therefore, the purpose of this study was 
to determine whether cognitive circuits are limited and whether alternate neural circuits are used for steering of gait. To address this aim, we used ${ }^{18} \mathrm{~F}$-FDG PET and an upright gait paradigm to measure rCGM in PD with and without FOG. We hypothesized that PD with FOG would have deficits in executive control, with limited recruitment of the cognitive corticobasal ganglia-thalamocortical circuit and increased activation of compensatory motor circuits.

\section{MATERIALS AND METHODS}

\section{Subjects}

The study protocol was approved by the McGill Faculty of Medicine Institutional Review Board for Human Subjects, and written informed consent was obtained from the patients.

Eighteen participants who had idiopathic PD according to the U.K. Brain Bank criteria and were able to walk independently for $30 \mathrm{~min}$ were recruited through the Quebec Parkinson Network (22). A score of 1 or greater in part I of the New Freezing-of-Gait Questionnaire was used to confirm 9 participants as experiencing FOG (FOG+) (23). We recruited 9 PD patients matched for age, sex, disease severity, disease duration, medication dosage, laterality of motor symptoms, and cognitive function who did not experience FOG (FOG-), as confirmed by a score of less than 1 in part I of the New Freezing-of-Gait Questionnaire (Table 1). All participants were free from cognitive impairment as assessed by the Montreal Cognitive Assessment ( $>25)$, had no coexisting orthopedic or neurologic disorders, and were nondiabetic (24).

\section{Experimental Procedure}

Participants were screened in their on-medication state. Disease severity was assessed with the Movement Disorders Society Unified Parkinson's Disease Rating Scale, part III, and the Hoehn and Yahr
Scale (25), and the assessment was repeated in the off-medication state later. The FOG+ group was further assessed for severity of FOG and its effect on daily life using parts II and III of the New Freezing-of-Gait Questionnaire, which included 8 additional questions resulting in a total score of 28 , with a higher score indicating more severe FOG. The Hospital Anxiety and Depression Scale was also applied (26). During this session, all participants briefly practiced the 2 gait tasks.

Two subsequent visits to the laboratory were made while the patients were in the clinically defined off-medication state (i.e., overnight withdrawal of all anti-Parkinson medication; average time off medication, $12 \pm 2 \mathrm{~h}$ ). Cerebral glucose metabolism was measured during 2 gait tasks, steering (i.e., complex locomotion) and straight walking (i.e., a simple locomotor reference task), using PET imaging with ${ }^{18} \mathrm{~F}$-FDG. Each task was performed continuously for $30 \mathrm{~min}$ immediately after a $185-\mathrm{MBq}$ bolus injection of ${ }^{18} \mathrm{~F}$-FDG on 2 separate occasions, at least $48 \mathrm{~h}$ apart (20). Participants walked at their self-selected normal walking speed and practiced each gait task approximately $10 \mathrm{~min}$ immediately before the tracer injection. The order of task performed was randomized across testing days. All subjects fasted overnight before both sessions $(\leq 6 \mathrm{~h})$.

For the walking tasks, 3 lanes (1.2-m width by $28-\mathrm{m}$ length) were delineated by yellow and orange cones in a $6-\mathrm{m}$ by $34-\mathrm{m}$ area (Fig. 1). In the straight-walking task, participants were instructed to walk in the middle of each lane, making $180^{\circ}$ turns into the adjacent lane (Fig. 1). In the steering task, the same placement of cone markers was used and participants were instructed to continuously turn around the yellow cones, placed in an unpredictable pattern. A safety harness was worn and a research assistant followed behind to prevent a fall (27). No falls occurred during the experiments. After the walking task, participants were escorted to the PET scanner from the experimental room, approximately a 5-min walk for all subjects. Therefore, PET scanning began within $50 \mathrm{~min}$ of the tracer injection. This walk to the scanner

TABLE 1

Subject Demographics

\begin{tabular}{|c|c|c|c|}
\hline Variable & $\mathrm{FOG}+(n=9)$ & FOG- $(n=9)$ & $P$ \\
\hline $\operatorname{Sex}(n)$ & & & 0.066 \\
\hline Male & 5 & 8 & \\
\hline Female & 4 & 1 & \\
\hline Age (y) & $68 \pm 6$ & $65 \pm 5$ & 0.235 \\
\hline Time since disease onset $(y)^{\star}$ & $9 \pm 6$ & $8 \pm 3$ & 0.863 \\
\hline Laterality of predominant motor symptoms $(n)$ & & & 0.500 \\
\hline Right & 3 & 2 & \\
\hline Left & 6 & 7 & \\
\hline Hoehn and Yahr Scale* & $3 \pm 1$ & $2 \pm 0$ & 0.258 \\
\hline MDS-UPDRSIII score (off drug) & $48 \pm 8$ & $41 \pm 7$ & 0.064 \\
\hline DOPA equivalent dose (mg) & $893 \pm 617$ & $751 \pm 272$ & 0.557 \\
\hline NFOG Questionnaire score* & $13 \pm 8$ & $0 \pm 0$ & $<0.001$ \\
\hline Montreal Cognitive Assessment & $28 \pm 2$ & $29 \pm 2$ & 0.321 \\
\hline \multicolumn{4}{|l|}{ Hospital Anxiety and Depression Scale } \\
\hline Anxiety & $6 \pm 3$ & $4 \pm 2$ & 0.067 \\
\hline Depression & $7 \pm 4$ & $4 \pm 2$ & 0.103 \\
\hline $\begin{array}{l}{ }^{*} \text { Nonparametric tests used. } \\
\text { MDS-UPDRSIII }=\text { Movement Disorders Society } \\
\text { Data are mean } \pm \text { SD for all variables except sex }\end{array}$ & $\begin{array}{l}\text { son's Disease R } \\
\text { represented as }\end{array}$ & $\begin{array}{l}\text { art III. } \\
\text { rquartile range. }\end{array}$ & \\
\hline
\end{tabular}




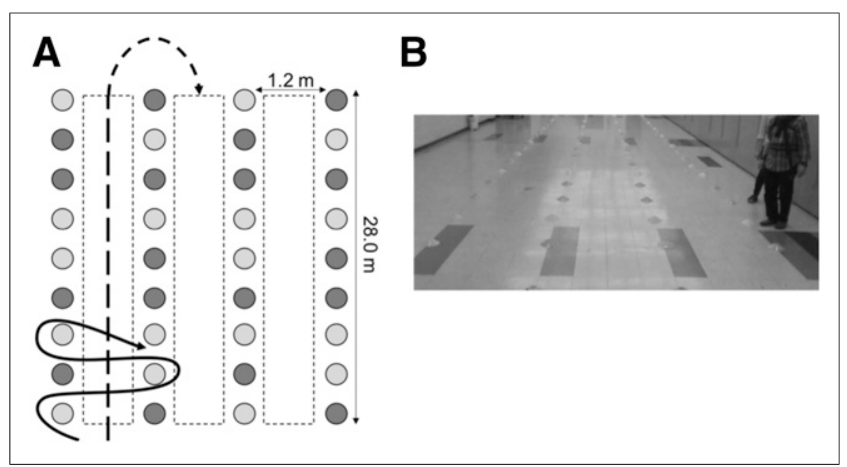

FIGURE 1. Experimental setup. (A) Solid line illustrates steering trajectory, and dashed line depicts straight walking. Light gray circles represent yellow cones, and dark gray circles represent orange cones. Complete experimental setup had 30 cones spanning entire length. (B) Participant performing steering task with experimenter following behind.

does not change ${ }^{18} \mathrm{~F}-\mathrm{FDG}$ concentration because it is static after the radiotracer uptake period of approximately $20 \mathrm{~min}(20,21)$.

Spatiotemporal measures of gait (i.e., stride length normalized to height and stride velocity) were measured using the APDM mobility lab system (Opal; APDM Inc.). Participants wore 6 wireless inertial sensors containing a triaxial accelerometer, triaxial gyroscope, and triaxial magnetometer. Data were sampled at $128 \mathrm{~Hz}$.

The number of FOG episodes and the total duration spent in FOG over the entire $30 \mathrm{~min}$ were evaluated using a stopwatch. Onset was defined as shuffling of the steps with minimal forward movement, trembling of the legs with absence of forward movement, or complete motor arrest (28). The same observer measured FOG across all participants. A video recording of the complete walking trial validated the number and duration of FOG episodes rated by another researcher masked to participant group. There was a high level of agreement between raters (number of episodes: $r=0.98, P<0.001$; total duration: $r=0.91, P<0.001$ ). Video-based analysis was used to determine the total distance walked.

\section{Imaging Protocol}

PET images were acquired on a High Resolution Research Tomograph PET scanner (CTI/Siemens) with a spatial resolution of 2.3$3.4 \mathrm{~mm}$ in full width at half maximum. Eight 5-min 3-dimensional sonograms were generated from list-mode data acquired over $40 \mathrm{~min}$. Normalization and correction of motion artifacts, random events, and scatter were applied before summation into a single 40-min frame. A 10-min transmission scan was acquired for attenuation correction.

Contiguous sagittal T1-weighted images were acquired on a Prisma 3-T scanner (Siemens) using a 3-dimensional magnetization-prepared rapid gradient-echo sequence (voxel size, $1 \mathrm{~mm}^{3}$; echo time, $2.96 \mathrm{~ms}$; repetition time, $2.3 \mathrm{~s}$; flip angle, $9^{\circ}$; number of slices, 192; slice thickness, $1 \mathrm{~mm}$; field of view, $256 \mathrm{~mm}^{2}$ ).

\section{Image Analysis}

Statistical parametric mapping software (SPM12; Wellcome Department of Cognitive Neurology) implemented in MATLAB R2015a (MathWorks) was used for image processing and statistical analysis. Processing was consistent with previously described methods (21). Briefly, reconstructed PET images were coregistered to each subjects' anatomic image and spatially normalized to the Montreal Neurological Institute template. Images were smoothed with a gaussian filter $(8 \mathrm{~mm}$ in full width at half maximum), and each voxel was scaled in proportion to the global mean activity, thus yielding estimates of relative rCGM.

rCGM during steering was directly compared with rCGM during straight walking to determine task-related activations for both groups.
To test an a priori hypothesis of FOG within the corticobasal gangliathalamocortical circuitry, statistical analysis was performed on regions of interest within the cognitive, motor, and limbic corticobasal ganglia-thalamocortical circuits (Supplemental Table 1; supplemental materials are available at http://jnm.snmjournals.org) $(6,13,15)$. The MarsBar toolbox in SPM was used to extract parameter estimates for each region of interest, which were then imported to SPSS (version 21.0, IBM) for further analysis by a 2-way ANOVA to determine the effect of task (repeated measures) and group. Second, whole-brain voxelwise analysis was performed using a flexible factorial design including subject, group, and task as factors. The main effects of task (steering vs. straight walking) and group $(\mathrm{FOG}+\mathrm{vs} . \mathrm{FOG}-$ ) and their interaction were determined at an uncorrected $P$ value of less than 0.005 and a cluster extent threshold of 30 voxels $(21,29)$.

\section{Statistical Analysis}

A 2-way ANOVA implemented in SPSS assessed the effect of task (repeated measures) and group (FOG + vs. $\mathrm{FOG}-$ ) on stride length and stride velocity. Post hoc tests were performed whenever a significant interaction occurred. Independent $t$ tests were used to assess group differences in clinical variables. When variables did not meet the assumption of normality assessed by a Shapiro-Wilk test, nonparametric Mann-Whitney $U$ tests were used. For the FOG+ group, the Pearson correlation coefficient $(r)$ was used to determine the relationship between peak activation in significant regions of interest with freezing severity and stride length, significant at a $P$ level of less than 0.05 (2-tailed). The Spearman rank-order correlation coefficient $\left(r_{\mathrm{s}}\right)$ was used for nonparametric data as indicated.

\section{RESULTS}

\section{Behavioral Outcomes}

Eight of 9 participants in the FOG + group experienced at least one FOG episode during the steering task, whereas only 3 participants experienced a FOG episode during straight walking. During steering, the median and interquartile range of the number of episodes was $5 \pm 1$, total time freezing was $5.22 \pm 31.60 \mathrm{~s}$, and the percentage of time spent freezing was $2.9 \% \pm 1.7 \%$. No episodes were observed in the FOG- group for either task.

The FOG+ group walked a shorter total distance than the FOG - group in both tasks $(P<0.01)$. Across tasks (main effect of group), the FOG + group had reduced stride length (FOG + , $61.6 \% \pm 2.3 \%$ height; FOG,$- 70.7 \% \pm 2.5 \%$ height; $P<0.05)$ and stride velocity $(\mathrm{FOG}+, 0.93 \pm 0.04 \mathrm{~m} / \mathrm{s} ; \mathrm{FOG}-, 1.2 \pm 0.05 \mathrm{~m} / \mathrm{s}$; $P<0.01$ ) compared with the FOG - group. During steering (main effect of task), both groups similarly decreased their stride length (FOG,$+ 26.0 \% \pm 0.09 \%$; FOG,$- 26.9 \% \pm 0.06 \%$ ) and stride velocity (FOG,$+ 32.4 \% \pm 0.12 \%$; FOG,$- 28.8 \% \pm 0.04 \%$ ) compared with straight walking $(P<0.001)$.

\section{Region-of-Interest Analysis}

The region-of-interest analysis demonstrated different task-related changes in metabolism between groups in the cognitive corticothalamic circuit only (i.e., left posterior parietal cortex, right dorsolateral prefrontal cortex, and left thalamus) (group $\times$ task: $P<0.05$ ) (Supplemental Table 2). More specifically, the FOG - group showed increased activity in the posterior parietal cortex and decreased activity in the dorsolateral prefrontal cortex and thalamus for steering, whereas there was no significant change in the FOG+ group.

\section{Whole-Brain rCGM During Steering}

During steering, the most prominent activation (steering $>$ straight) in both groups was in the left superior parietal lobule and the inferior parietal lobule (Supplemental Table 3). The prominent 
deactivations (steering $<$ straight) were in the inferior frontal gyrus and thalamus. Task-related metabolic differences were observed between groups in several regions (Fig. 2; Supplemental Table 4). The FOG + group had increased activity during steering (steering $>$ straight) in the right cerebellum (Crus II), supplementary motor area, left superior medial gyrus, posterior-medial frontal gyrus, and temporal gyri (middle and superior) than the FOG - group. The FOG+ group also demonstrated more deactivation (steering $<$ straight) in the inferior frontal gyrus. In comparison, the FOG + group had reduced activation (steering $>$ straight) of the left superior and inferior parietal lobule, as well as the superior frontal gyrus, compared with the FOG - group. The FOG + group had less deactivation (steering $<$ straight) in the right frontal gyri (middle and inferior), left posterior medial frontal gyrus, precentral gyrus, superior frontal gyrus, and thalamus.

\section{Relationship Between rCGM, Disease Severity, and Gait Outcomes}

Increased metabolic activity in the right dorsolateral prefrontal cortex during steering (steering $>$ straight) was associated with reduced stride length in the FOG + group $(r=-0.71, P=0.033)$. This activity was not associated with clinical $\left(r_{\mathrm{s}}=-0.08, P=\right.$ 0.831 ) or objective (number of episodes: $r_{\mathrm{s}}=-0.03, P=0.931$; total duration: $r_{\mathrm{s}}=0.17, P=0.668$ ) measures of FOG severity. Activity in the posterior parietal cortex, thalamus, and supplementary motor area did not correlate with gait impairments or FOG severity. In the FOG - group, there were no correlations between rCGM and stride length for any regions of interest.

\section{DISCUSSION}

Changes in cerebral glucose metabolism associated with complex walking were measured in PD with and without FOG. In line with the hypothesis for executive dysfunction, we observed that the FOG + group had reduced modulation of metabolic activity in the cognitive corticothalamic circuit during steering of gait compared with straight walking (Fig. 3). More specifically, the FOG + group had less activation of the posterior parietal cortex

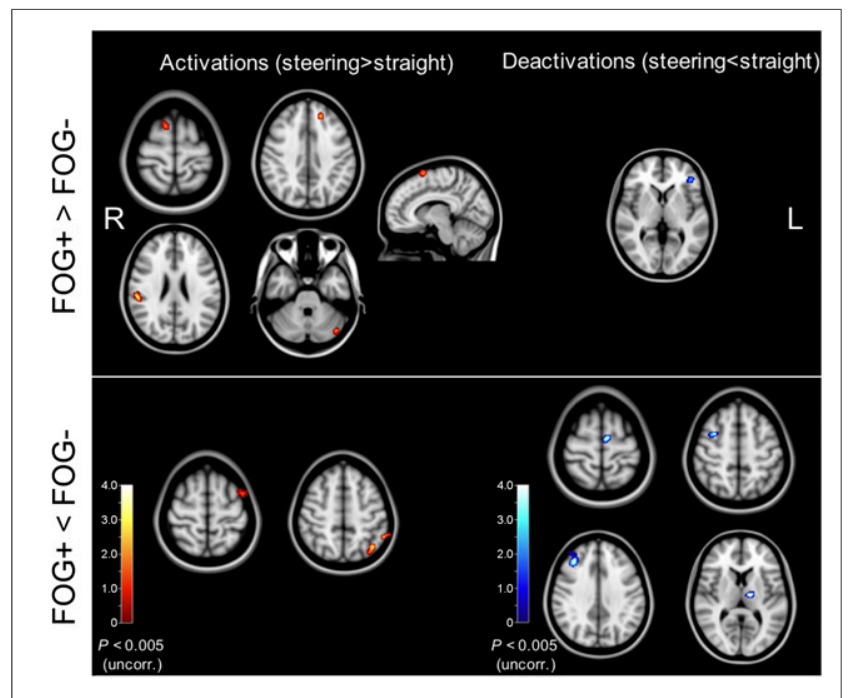

FIGURE 2. Statistical parametric maps showing steering-related rCGM group difference. Activations and deactivations are represented by warm and cool colors, respectively. $P<0.005$ (uncorrected); cluster extent threshold $=30$.

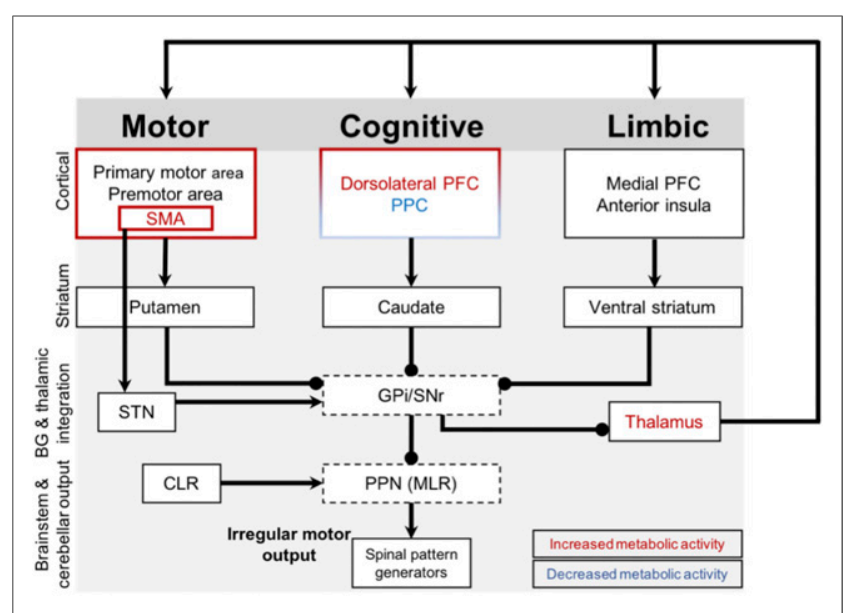

FIGURE 3. Complex locomotor control in FOG. Arrows indicate excitatory connections, and spheric ends denote inhibitory connections. Red and blue labels are regions with increased and decreased cerebral glucose metabolism, respectively, in FOG + group compared with FOGgroup. FOG + group demonstrates changed metabolic activity in cognitive corticobasal ganglia-thalamocortical circuitry (less activation of parietal and less deactivation of prefrontal cortices). At the same time, there is less deactivation of thalamus during steering and increased activity of supplementary motor area, known to have hyperdirect connections with subthalamic nucleus, with overall inhibitory effect on already impaired basal ganglia outputs (i.e., globus pallidus internal segment and substantia nigra) and brain stem locomotor nuclei (i.e., pedunculopontine nucleus). $B G=$ basal ganglia; $C L R=$ cerebellar locomotor region; $\mathrm{GPi}=$ globus pallidus internal segment; $\mathrm{PFC}=$ prefrontal cortex; PPC = posterior parietal cortex; PPN (MLR) = mesencephalic locomotor region; $\mathrm{SMA}=$ supplementary motor area; $\mathrm{SNr}=$ substantia nigra; STN = subthalamic nucleus.

and less deactivation of the dorsolateral prefrontal cortex and thalamus than the FOG - group. In addition, the FOG + group had increased activation in the supplementary motor area (medial superior frontal gyrus) and less deactivation in the mesial frontal (inferior, superior, posterior-medial) gyri during steering. Activity in the dorsolateral prefrontal cortex correlated with gait impairment (i.e., reduced stride length) in the $\mathrm{FOG}+$ group.

The frontal and parietal regions with changed glucose metabolism during steering in the FOG + group compose the cognitive corticothalamic circuitry and are importantly involved in mediating executive function (30) and visuomotor integration (31). It has recently been demonstrated that this network is active during lower limb motor arrests, thought to serve a positive compensatory strategy to break a FOG episode because individuals with severe FOG limit recruitment of this network (6). Structural (32) and functional $(5,33)$ neuroimaging findings also demonstrate that this network is globally impaired in individuals with FOG. Therefore, our results, which illustrate reduced activation of parietal regions and reduced deactivation of prefrontal regions within this network, could indicate that the FOG + group ineffectively activated parietal regions for steering of gait and used more prefrontal control than the FOG - group. Increased activation of the dorsolateral prefrontal cortex correlated with gait impairment (i.e., reduced stride length) in the FOG + group. This finding is interesting because during real FOG episodes there is increased activity of both prefrontal and parietal regions (6). Therefore, this shift from parietal to prefrontal control within the cognitive circuit illustrates a 
unique control mechanism used by the FOG + group during complex walking that may fail in a FOG event.

The supplementary motor area was increased in the FOG+ group for steering of gait. Notably, the supplementary motor area has direct projections to the subthalamic nucleus (i.e., hyperdirect pathway) responsible for strong inhibition of a planned action $(8,9)$ and is strongly activated during real FOG episodes $(34)$. In these events, excitation of the subthalamic nucleus by the supplementary motor area leads to inhibition of basal ganglia and brain stem output nuclei believed to result in a lack of locomotor output for successful gait. Thus, during steering, the FOG + group has increased activity within the hyperdirect pathway, known to result in inhibition of subcortical nuclei during FOG episodes. Taken together, these results provide evidence for a FOG mechanism that is active during increased cognitive and motor demands associated with complex walking in the FOG + group. The aforementioned parietal-to-prefrontal control shift observed in the present study could represent a compensatory strategy used during complex walking to prevent FOG episodes-a strategy that may become inefficient during motor arrest.

fMRI virtual reality used to study turning during continuous pedaling in FOG (i.e., between episodes) reveals increased activity of the inferior frontal regions and decreased activity of the parietal and supplementary motor areas (18). During steering, we also observed increased inferior frontal and reduced parietal metabolism in the FOG + group; however, our results demonstrate activation of the supplementary motor area. The present study measures global activation during a complex gait relative to straight walking and illustrates how-during an upright gait-individuals with FOG recruit circuitry different from that recruited by individuals without FOG.

Both groups showed reduced metabolic activity of the thalamus during steering; however, the FOG + group demonstrated less deactivation. Indeed, the thalamus and other subcortical nuclei subserve automatic motor output $(10,35)$. Furthermore, it has been suggested that there is reduced automaticity in PD with FOG, evoking increased cortical control (14). Indeed, steering of gait requires increased top-down control due to the integration of internal and external movement goals and the high degree of motor planning required, compared with simple steady-state walking (21). Therefore, reduced thalamic deactivation could demonstrate a poor ability to shift between automatic and voluntary control across simple and complex gait tasks in PD with FOG.

In contrast to our findings, a previous study in PD with and without FOG showed increased metabolic activity of parietal regions and subcortical nuclei, as well as reduced activity of frontal regions during an upright complex gait task (i.e., combined dual-tasking, passing through a narrow space, and $360^{\circ}$ turning) compared with supine rest (36). Notably, participants spent approximately $40 \%$ of the time in motor arrest. Moreover, the poor temporal resolution of PET and the combined gait task used in this study render the results difficult to interpret with respect to locomotor control. In comparison, our paradigm isolated complex gait from upright stance and steady-state gait and resulted in a small percentage of the total trial in motor arrest (average of $2.5 \%$ ). Therefore, our findings can be interpreted to better reflect mechanisms underlying complex walking, although they do not completely dissect these phenomena apart. Furthermore, our results do not demonstrate a significant role for the mesencephalic locomotor region in control of complex gait in PD with FOG. The mesencephalic locomotor region has previously been proposed as an integral structure in the pathophysiology of FOG because of its role in initiation and modulation of gait (37) and impairment in the FOG + group (5). Previous evidence using fMRI during continuous foot pedaling showed decreased activity of the mesencephalic locomotor region during FOG episodes, thought to occur from strong inhibition from the globus pallidus internus (6). In comparison, investigation of imagined gait using fMRI has shown conflicting results regarding the mesencephalic locomotor region's role in FOG, likely because of the discrepancy between actual and perceived gait in PD with FOG $(5,16)$. Moreover, our results suggest that the mesencephalic locomotor region may not be directly implicated in PD with FOG during upright complex walking as compared with straight walking. However, this result must be interpreted carefully, because it is possible that the present paradigm cannot detect ${ }^{18}$ F-FDG uptake in these brain stem nuclei.

\section{CONCLUSION}

To our knowledge, this is the first investigation of complex locomotor control in PD with FOG during real gait. Our findings demonstrate that PD with FOG has reduced parietal control and alternate control via prefrontal and supplementary motor cortices compared with PD without FOG during complex walking. Our results provide novel information about the neural mechanisms involved in FOG.

\section{DISCLOSURE}

This work was supported by Parkinson Canada. No other potential conflict of interest relevant to this article was reported.

\section{ACKNOWLEDGMENTS}

We thank the MRI and PET units of the McConnell Brain Imaging Centre.

\section{REFERENCES}

1. Giladi N, McDermott MP, Fahn S, et al. Freezing of gait in PD: prospective assessment in the DATATOP cohort. Neurology. 2001;56:1712-1721.

2. Spildooren J, Vercruysse S, Desloovere K, Vandenberghe W, Kerckhofs E, Nieuwboer A. Freezing of gait in Parkinson's disease: the impact of dual-tasking and turning. Mov Disord. 2010;25:2563-2570.

3. Mirelman A, Maidan I, Bernad-Elazari H, Shustack S, Giladi N, Hausdorff JM. Effects of aging on prefrontal brain activation during challenging walking conditions. Brain Cogn. 2017;115:41-46.

4. Lewis SJ, Shine JM. The next step: a common neural mechanism for freezing of gait. Neuroscientist. 2016;22:72-82.

5. Snijders AH, Leunissen I, Bakker M, et al. Gait-related cerebral alterations in patients with Parkinson's disease with freezing of gait. Brain. 2011;134:59-72.

6. Shine JM, Matar E, Ward PB, et al. Exploring the cortical and subcortical functional magnetic resonance imaging changes associated with freezing in Parkinson's disease. Brain. 2013;136:1204-1215.

7. Maidan I, Bernad-Elazari H, Gazit E, Giladi N, Hausdorff JM, Mirelman A. Changes in oxygenated hemoglobin link freezing of gait to frontal activation in patients with Parkinson disease: an fNIRS study of transient motor-cognitive failures. J Neurol. 2015;262:899-908.

8. Cavanagh JF, Wiecki TV, Cohen MX, et al. Subthalamic nucleus stimulation reverses mediofrontal influence over decision threshold. Nat Neurosci. 2011;14: 1462-1467.

9. Aron AR, Poldrack RA. Cortical and subcortical contributions to stop signal response inhibition: role of the subthalamic nucleus. J Neurosci. 2006;26:24242433.

10. Vandenbossche J, Deroost N, Soetens E, et al. Freezing of gait in Parkinson's disease: disturbances in automaticity and control. Front Hum Neurosci. 2013;6:356.

11. Dagan M, Herman T, Harrison R, et al. Multitarget transcranial direct current stimulation for freezing of gait in Parkinson's disease. Mov Disord. 2018;33: $642-646$. 
12. Fling BW, Cohen RG, Mancini M, et al. Functional reorganization of the locomotor network in Parkinson patients with freezing of gait. PLoS One. 2014;9: e100291.

13. Lewis SJ, Barker RA. A pathophysiological model of freezing of gait in Parkinson's disease. Parkinsonism Relat Disord. 2009;15:333-338.

14. Hallett M. The intrinsic and extrinsic aspects of freezing of gait. Mov Disord. 2008;23(suppl 2):S439-S443.

15. Alexander GE, DeLong MR, Strick PL. Parallel organization of functionally segregated circuits linking basal ganglia and cortex. Annu Rev Neurosci. 1986;9: 357-381.

16. Peterson DS, Pickett KA, Duncan R, Perlmutter J, Earhart GM. Gait-related brain activity in people with Parkinson disease with freezing of gait. PLoS One. 2014;9:e90634.

17. Cohen RG, Chao A, Nutt JG, Horak FB. Freezing of gait is associated with a mismatch between motor imagery and motor execution in narrow doorways, not with failure to judge doorway passability. Neuropsychologia. 2011;49:3981-3988.

18. Gilat M, Shine JM, Walton CC, O'Callaghan C, Hall JM, Lewis SJG. Brain activation underlying turning in Parkinson's disease patients with and without freezing of gait: a virtual reality fMRI study. NPJ Parkinsons Dis. 2015;1:15020.

19. Horak FB. Postural orientation and equilibrium: what do we need to know about neural control of balance to prevent falls? Age Ageing. 2006;35(suppl 2):ii7-ii11.

20. la Fougère $\mathrm{C}$, Zwergal $\mathrm{A}$, Rominger $\mathrm{A}$, et al. Real versus imagined locomotion: a [ ${ }^{18}$ F]-FDG PET-fMRI comparison. Neuroimage. 2010;50:1589-1598.

21. Mitchell T, Starrs F, Soucy JP, Thiel A, Paquette C. Impaired sensorimotor processing during complex gait precedes behavioral changes in middle-aged adults. J Gerontol A Biol Sci Med Sci. September 21, 2018 [Epub ahead of print].

22. Hughes AJ, Daniel SE, Kilford L, Lees AJ. Accuracy of clinical diagnosis of idiopathic Parkinson's disease: a clinico-pathological study of 100 cases. J Neurol Neurosurg Psychiatry. 1992;55:181-184.

23. Nieuwboer A, Rochester L, Herman T, et al. Reliability of the new freezing of gait questionnaire: agreement between patients with Parkinson's disease and their carers. Gait Posture. 2009;30:459-463.

24. Nasreddine ZS, Phillips NA, Bedirian V, et al. The Montreal Cognitive Assessment, MoCA: a brief screening tool for mild cognitive impairment. J Am Geriatr Soc. 2005;53:695-699.
25. Goetz CG, Fahn S, Martinez-Martin P, et al. Movement Disorder Society-sponsored revision of the Unified Parkinson's Disease Rating Scale (MDS-UPDRS): process, format, and clinimetric testing plan. Mov Disord. 2007;22:41-47.

26. Zigmond AS, Snaith RP. The hospital anxiety and depression scale. Acta Psychiatr Scand. 1983;67:361-370.

27. Paquette C, Franzen E, Jones GM, Horak FB. Walking in circles: navigation deficits from Parkinson's disease but not from cerebellar ataxia. Neuroscience. 2011;190:177-183.

28. Schaafsma JD, Balash Y, Gurevich T, Bartels AL, Hausdorff JM, Giladi N. Characterization of freezing of gait subtypes and the response of each to levodopa in Parkinson's disease. Eur J Neurol. 2003;10:391-398.

29. Zwergal A, Schoberl F, Xiong G, et al. Anisotropy of human horizontal and vertical navigation in real space: behavioral and PET correlates. Cereb Cortex. 2016;26:4392-4404.

30. Spreng RN, Stevens WD, Chamberlain JP, Gilmore AW, Schacter DL. Default network activity, coupled with the frontoparietal control network, supports goaldirected cognition. Neuroimage. 2010;53:303-317.

31. Battaglia-Mayer A, Caminiti R, Lacquaniti F, Zago M. Multiple levels of representation of reaching in the parieto-frontal network. Cereb Cortex. 2003;13: 1009-1022.

32. Tessitore A, Amboni M, Cirillo G, et al. Regional gray matter atrophy in patients with Parkinson disease and freezing of gait. AJNR Am J Neuroradiol. 2012;33: 1804-1809.

33. Tessitore A, Amboni M, Esposito F, et al. Resting-state brain connectivity in patients with Parkinson's disease and freezing of gait. Parkinsonism Relat Disord. 2012;18:781-787.

34. Shine JM, Handojoseno AM, Nguyen TN, et al. Abnormal patterns of theta frequency oscillations during the temporal evolution of freezing of gait in Parkinson's disease. Clin Neurophysiol. 2014;125:569-576.

35. Jahn K, Deutschlander A, Stephan T, et al. Supraspinal locomotor control in quadrupeds and humans. Prog Brain Res. 2008;171:353-362.

36. Tard C, Delval A, Devos D, et al. Brain metabolic abnormalities during gait with freezing in Parkinson's disease. Neuroscience. 2015;307:281-301.

37. Jahn K, Deutschlander A, Stephan T, et al. Imaging human supraspinal locomotor centers in brainstem and cerebellum. Neuroimage. 2008;39:786-792. 